\title{
Nonspherical dust grains in mean-motion orbital resonances
}

\author{
M. Kocifaj ${ }^{1}$ and J. Klačka ${ }^{2}$ \\ 1 Astronomical Institute, Slovak Academy of Sciences, Dúbravská cesta 9, 84504 Bratislava, Slovak Republic \\ e-mail: astromir@savba.sk \\ 2 Department of Astronomy, Physics of the Earth, and Meteorology, Faculty of Mathematics, Physics and Informatics, Comenius \\ University, Mlynská dolina, 84248 Bratislava, Slovak Republic \\ e-mail: klacka@fmph.uniba.sk
}

Received 7 August 2007 / Accepted 8 February 2008

ABSTRACT

\begin{abstract}
The effect of stellar electromagnetic radiation on the motion of arbitrarily shaped dust particles in mean-motion orbital resonances with planets is investigated. Giving a resonance and a value of parameter $\beta$ (the ratio between radiation pressure force and gravitational force of the central star), nonradial components of radiation pressure force are calculated when a given trajectory is prescribed. The found values of the nonradial components are in a good agreement with published detailed numerical simulations for a given nonspherical particle. Therefore nonspherical grains are present in mean-motion resonances with planets. Similarly, the difference between the motion of a real particle and the motion influenced by the Poynting-Robertson effect may be important.
\end{abstract}

Key words. scattering - celestial mechanics - meteors, meteoroids - interplanetary medium

\section{Introduction}

The motion of dust grains near mean-motion orbital resonances with planets has been systematically investigated since the paper by Jackson \& Zook (1989). The authors considered the motion of spherical interplanetary dust grains near mean-motion orbital resonances with the Earth. Many papers have considered this situation, e.g., Weidenschilling \& Jackson (1993), Beaugé \& Ferraz-Mello (1994), Marzari \& Vanzani (1994), Šidlichovský \& Nesvorný (1994), Liou \& Zook (1995), Liou et al. (1995), Liou \& Zook (1997). One of the results of these works was that dust rings around planetary orbits should exist. This was found and the observational situation is described in papers by Dermott et al. (1994), Brownlee (1994), Reach et al. (1995).

All the above presented theoretical papers considered the Poynting-Robertson effect as the effect of solar electromagnetic radiation acting on the motion of dust grains. However, the Poynting-Robertson effect holds for spherical particles only. Since real dust particles are arbitrarily shaped (Wurm \& Schnaiter 2002), theoretical models have to be generalized to nonspherical particles.

The first paper concerning action of electromagnetic radiation on nonspherical particle was written by Einstein more than a hundred years ago (Einstein 1905). However, the first equation of motion, containing also Einstein's case and the PoyntingRobertson effect, was given by Klačka (1994) and in terms of optical properties by Klačka \& Kocifaj (1994) (see also Kocifaj et al. 2000). Klačka (2000, 2004) presented relativistically covariant equation of motion for arbitrarily shaped dust grains. The fact that a nonspherical particle moves in a different way than a spherical one was confirmed experimentally by Krauss \& Wurm (2004). Detail numerical simulations for the orbital evolution of nonspherical dust grains near mean-motion orbital resonances with a planet was discussed in Klačka et al. (2005) and in Klačka \& Kocifaj (2006a). The papers found that nonspherical dust grains also can be captured into the mean-motion orbital resonances with a planet. Moreover, the papers found the first case when a physically justified force can generate resonant trapping for diverging orbits (the secular evolution of the semimajor axis of the particle is an increasing function of time before capture into a resonance).

This paper deals with the effect of stellar (solar) electromagnetic radiation on the motion of an arbitrarily shaped dust particle. We consider the orbital evolution of dust grains near meanmotion orbital resonances with Neptune (the resonant captures of nonspherical particles were published only for Neptune). The motion of interplanetary dust grains around the Sun is under the action of gravity of the Sun and Neptune and solar electromagnetic radiation: the effect is dominant for the grains of radii between $1 \mu \mathrm{m}$ and $100 \mu \mathrm{m}$ (Leinert \& Grün 1990) and we consider particles of radii within this interval. However, instead of detailed numerical calculations for a particle of a given shape and optical properties, we are interested in finding characteristics of nonspherical particles when their trajectories are prescribed as trajectories near a given mean-motion orbital resonance. Mainly, we are interested in nonradial components of the radiation force, in the proper frame of the particle, which enable trajectories near motion-orbital resonances; the obtained results will be compared with the direct task solved by Klačka et al. (2005) and Klačka \& Kocifaj (2006a).

\subsection{Equation of motion: radiation and gravity}

We are interested in orbital evolution near mean-motion orbital resonances with a planet in the gravitational field of the central star. We will consider the Sun as the central star. If we do not consider the radiation pressure component normal to the orbital plane of the planet - the normal component is of marginal importance for the existence and character of the motion near meanmotion orbital resonances (Klačka et al. 2005; Klačka \& Kocifaj 2006a), then the equation of motion, if we do not consider the 
emission component of the radiation force, is:

$$
\begin{aligned}
& \frac{\mathrm{d} \boldsymbol{v}}{\mathrm{d} t}=-\frac{G M_{\odot}}{r^{3}} \boldsymbol{r}+\beta \frac{G M_{\odot}}{r^{2}} \\
& \times\left\{\left(1-\frac{\boldsymbol{v} \cdot \boldsymbol{e}_{R}}{c}\right) \boldsymbol{e}_{R}-\frac{\boldsymbol{v}}{c}+\frac{\beta_{2}}{\beta} \boldsymbol{e}_{T}\right\} \\
&-G m_{\mathrm{P}}\left\{\frac{\boldsymbol{r}-\boldsymbol{r}_{\mathrm{P}}}{\left|\boldsymbol{r}-\boldsymbol{r}_{\mathrm{P}}\right|^{3}}+\frac{\boldsymbol{r}_{\mathrm{P}}}{\left|\boldsymbol{r}_{\mathrm{P}}\right|^{3}}\right\}, \\
& \boldsymbol{e}_{R} \equiv \boldsymbol{r} /|\boldsymbol{r}|, \\
& \boldsymbol{e}_{T} \cdot \boldsymbol{e}_{R}=0, \\
& \beta \equiv \frac{L_{\odot} \pi R^{2}}{4 \pi G M_{\odot} m} \frac{\bar{Q}_{\mathrm{pr}, 1}^{\prime}}{c} \\
&= 7.6 \times 10^{-4} \bar{Q}_{\mathrm{pr}, 1}^{\prime} \frac{\pi R^{2}\left[\mathrm{~m}^{2}\right]}{m[\mathrm{~kg}]}, \\
& \beta_{2} \equiv \frac{L_{\odot} \pi R^{2}}{4 \pi G M_{\odot} m} \frac{\bar{Q}_{\mathrm{pr}, 2}^{\prime}}{c} \\
&= 7.6 \times 10^{-4} \bar{Q}_{\mathrm{pr}, 2}^{\prime} \frac{\pi R^{2}\left[\mathrm{~m}^{2}\right]}{m[\mathrm{~kg}]},
\end{aligned}
$$

where $G$ is the gravitational constant, $M_{\odot}$ is mass of the Sun, $r$ is the position vector of the particle with respect to the Sun, $r=|\boldsymbol{r}|$, $\boldsymbol{r}_{\mathrm{P}}$ is the position vector of the planet with respect to the Sun, $m_{\mathrm{P}}$ is mass of the planet, $m$ is mass of the particle and $R$ is its effective radius, $\bar{Q}_{\mathrm{pr}, 1}^{\prime}$ is the efficiency factor for the radiation pressure integrated over the solar spectrum and calculated for the radial direction (as for a dimensionless factor of the effectiveness of radiation pressure see also, e.g., Mie 1908, or, Sect. 4.5 in Bohren \& Huffman 1983, Eqs. (21)-(23) in Klačka \& Kocifaj 2001, Eqs. (2)-(5) in Klačka et al. 2006; compare with the geometrical approach in Eqs. (1), (2), (9), ... in Makuch et al. 2006). We have also used that the flux density of radiative energy is $L_{\odot} /\left(4 \pi r^{2}\right)$, where $L_{\odot}$ is the solar luminosity. $\bar{Q}_{\mathrm{pr}, 2}^{\prime}$ is efficiency factor for radiation pressure in the direction $\boldsymbol{e}_{T}$ (see Klačka et al. 2006). We consider the effect of the electromagnetic force to the first order in $\boldsymbol{v} / c$, where $\boldsymbol{v}$ is the heliocentric velocity of the particle and $c$ is the speed of light. As for the radiation term in Eq. (7), the case $\beta_{2} \equiv 0$ corresponds to the Poynting-Robertson effect, which holds for spherical particles (Robertson 1937; Klačka 1992). The radiation term in Eq. (7) follows from Eq. (1) by putting $F_{e j}^{\prime} \equiv$ $0, j=1$ to 3 , and $\bar{Q}_{3}^{\prime} \equiv 0$. We will suppose that the planet moves in a circular orbit around the Sun.

Now, we will make an approximation that the motion of the particle is driven mainly by gravitational effect of the Sun - an iterative procedure for which the radial case is the first approximation. We will use the following equations known from the two-body problem:

$$
\begin{aligned}
\boldsymbol{v} & =v_{R} \boldsymbol{e}_{R}+v_{T} \boldsymbol{e}_{T}, \\
v_{R} & =\sqrt{\frac{G M_{\odot}(1-\beta)}{a_{\mathrm{res}}\left(1-e^{2}\right)}} e \sin (\Theta-\omega), \\
v_{T} & =\sqrt{\frac{G M_{\odot}(1-\beta)}{a_{\mathrm{res}}\left(1-e^{2}\right)}}\{1+e \cos (\Theta-\omega)\}, \\
\boldsymbol{e}_{R} & \equiv(\cos \Theta, \sin \Theta), \\
\boldsymbol{e}_{T} & \equiv(-\sin \Theta, \cos \Theta), \\
\frac{\mathrm{d} \boldsymbol{e}_{R}}{\mathrm{~d} t} & =\boldsymbol{e}_{T} \frac{\mathrm{d} \Theta}{\mathrm{d} t},
\end{aligned}
$$

$$
\begin{aligned}
& \frac{\mathrm{d} \boldsymbol{e}_{T}}{\mathrm{~d} t}=-\boldsymbol{e}_{R} \frac{\mathrm{d} \Theta}{\mathrm{d} t}, \\
& \frac{\mathrm{d} \boldsymbol{v}}{\mathrm{d} t}=-\frac{G M_{\odot}(1-\beta)}{r^{3}} \boldsymbol{r},
\end{aligned}
$$

where $a_{\text {res }}$ is the resonant semi-major axis of the particle, eccentricity $e$ is considered to be almost constant during several revolutions around the Sun and $\beta$ is considered to be slowly changing; true anomaly is given by the relation $\Theta-\omega$. The resonant semi-major axis is

$$
\begin{aligned}
& a_{\mathrm{res}}=a_{\mathrm{P}}(1-\beta)^{1 / 3}\left(\frac{n_{\mathrm{P}}}{n}\right)^{2 / 3}, \\
& \frac{n_{\mathrm{P}}}{n}=\frac{p+q}{p},
\end{aligned}
$$

where $n_{\mathrm{P}}$ and $n$ are mean motions of the planet (with semi-major axis $a_{\mathrm{P}}$ is the radius of the circle, in our case) and of the particle, $p$ and $q$ are small natural numbers defining the type of resonance.

Inserting Eqs. (2) into (1), we obtain

$$
\begin{aligned}
\beta_{2} \boldsymbol{e}_{T}-\beta\left(\frac{\boldsymbol{v} \cdot \boldsymbol{e}_{R}}{c} \boldsymbol{e}_{R}+\frac{\boldsymbol{v}}{c}\right)= & \frac{m_{\mathrm{P}}}{M_{\odot}}|\boldsymbol{r}|^{2} \\
& \times\left\{\frac{\boldsymbol{r}-\boldsymbol{r}_{\mathrm{P}}}{\left|\boldsymbol{r}-\boldsymbol{r}_{\mathrm{P}}\right|^{3}}+\frac{\boldsymbol{r}_{\mathrm{P}}}{\left|\boldsymbol{r}_{\mathrm{P}}\right|^{3}}\right\},
\end{aligned}
$$

and the following equations also will be used:

$r_{\mathrm{P}}=a_{\mathrm{P}}\left\{\cos \left(\omega_{\mathrm{P}} t+\varphi_{0}\right), \sin \left(\omega_{\mathrm{P}} t+\varphi_{0}\right)\right\}$,

$\omega_{\mathrm{P}} \equiv \sqrt{\frac{G M_{\odot}}{a_{\mathrm{P}}^{3}}}$,

$|\boldsymbol{r}|=a_{\mathrm{res}}\left(1-e^{2}\right) /\{1+e \cos (\Theta-\omega)\}$,

$\frac{\mathrm{d} \Theta}{\mathrm{d} t}=\sqrt{\frac{G M_{\odot}(1-\beta)}{\left[a_{\mathrm{res}}\left(1-e^{2}\right)\right]^{3}}}\{1+e \cos (\Theta-\omega)\}^{2}$,

where $t$ is time and $\varphi_{0}$ denotes the initial position $(t=0)$ of the planet. For the secular evolution of eccentricity, we use the following ansatz:

$e=e_{\text {in }}+\frac{e_{\text {lim }}-e_{\text {in }}}{T_{\text {res }}} t$,

where $e_{\text {in }}$ is the initial eccentricity (a starting value) and we take $T_{\text {res }}=5 \times 10^{4} \mathrm{yrs}$, as an example. The relation (12) is an approximation both for spherical and nonspherical particles. The asymptotic eccentricity $e_{\lim }$ is given by the following equation

$\frac{n_{\mathrm{P}}}{n}=\frac{1+3 e_{\lim }^{2}}{\left(1-e_{\lim }^{2}\right)^{3 / 2}}$,

see Liou \& Zook (1997), Klačka \& Kocifaj (2006b - Eq. (83)).

\section{Numerical results}

We are interested mainly in values of $\beta_{2}$ for the situation when a particle is moving near a mean-motion orbital resonance with Neptune. As an approximation, we consider orbital elements of the particle to be constants, only secular evolution of the eccentricity is prescribed by Eqs. (3), (6), (7). In order to allow more realistic motion than Keplerian motion, we allow the value of $\beta$ to change: the change of $\beta$ is less than $20 \%$ of its mean value. As a consequence, we have obtained various results for the density 


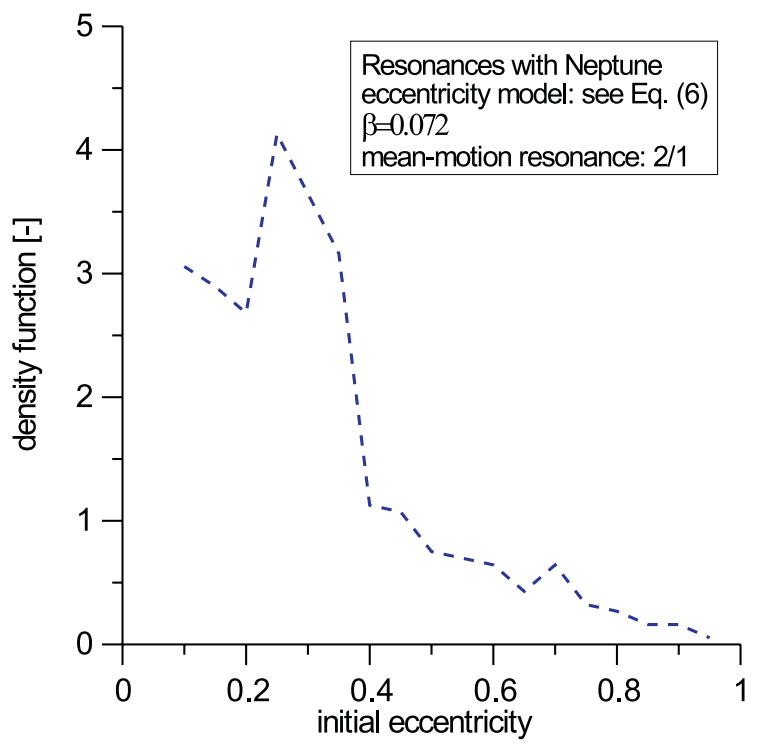

Fig. 1. The normalized density function reflecting the probability distribution of the particles moving in accordance with Eq. (6), where $e_{\text {in }}$ varies from 0.10 to 0.95 . The particles are captured in the $2 / 1$ resonance. The exact value of $\beta=0.072$ was chosen to compare with the former results (Klačka \& Kocifaj 2006a).

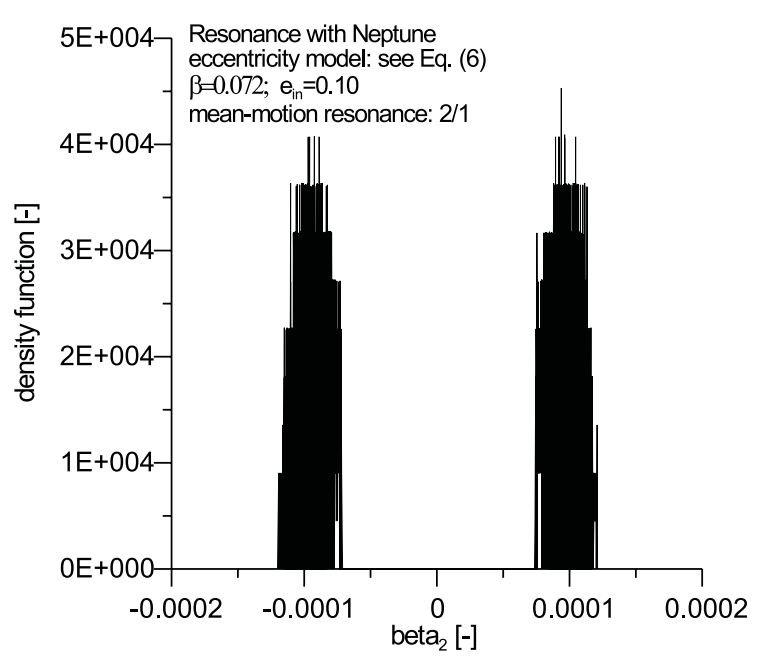

Fig. 2. Density function for $\beta_{2}$ for the case $e_{\text {in }}=0.10$.

functions for $\beta_{2}, \beta$, eccentricity of the particle and $\Theta_{0}-\varphi_{0}$ (relative initial position of the particle with respect to the planet) for the cases when the prescribed orbital evolution yields values of $\beta$ in a given interval $(1.0 \pm 0.2) \times \beta$ (mean).

The calculations for $\beta=0.072$ have shown that particles captured in the resonance $2 / 1$, evolving in agreement with Eq. (6), will be accumulated predominately at slightly eccentric orbits. The number density of the particles peaks at about $e_{\text {in }} \approx 0.2-0.3$ (Fig. 1) and the capture probability rapidly decreases as initial eccentricity $e_{\text {in }}$ approaches 1 .

The detailed behavior of the particles at different orbits is documented in Figs. 2-4, where density functions for $\beta_{2}$ at three different eccentricities $\left(e_{\text {in }}=0.10, e_{\text {in }}=0.25\right.$, and $\left.e_{\text {in }}=0.75\right)$ are depicted. At small eccentricities the density function is formed into a bimodal distribution with accumulation modes situated near $\beta_{2}=-1 \times 10^{-4}$ and $\beta_{2}=+1 \times$ $10^{-4}$. The width of both peaks increases with growing $e_{\text {in }}$. The bimodality of the density function disappears at large initial

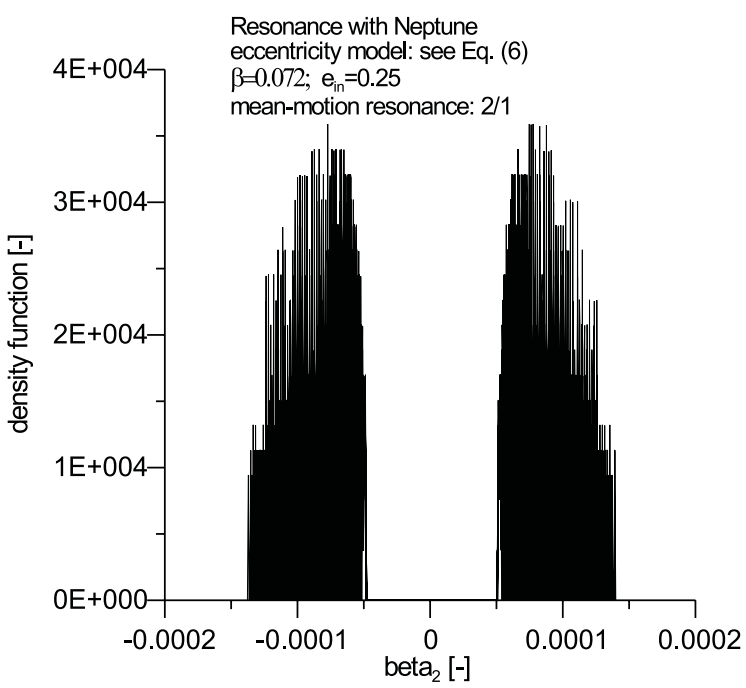

Fig. 3. Density function for $\beta_{2}$ for the case $e_{\text {in }}=0.25$.

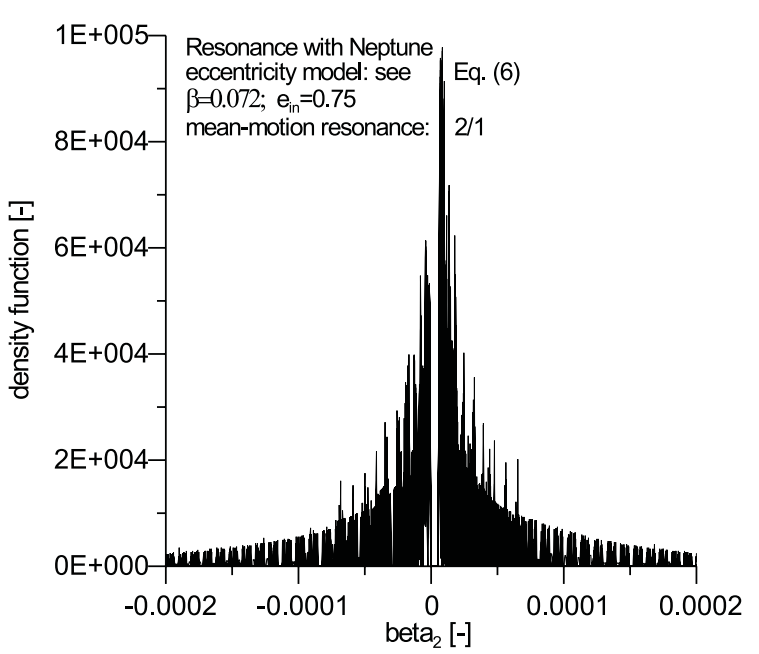

Fig. 4. Density function for $\beta_{2}$ for the case $e_{\text {in }}=0.75$.

eccentricities. Normally a wide spread of $\beta_{2}$ is found when one deals with populations of particles moving at highly eccentric orbits (with $e_{\text {in }}>0.75$ ). In such a case the density function typically peaks at about $\beta_{2} \approx 0$ (note that the ratio of $\beta_{2} / \beta$ becomes important when it is comparable to $v / c$, i.e., when $\beta_{2} / \beta \approx$ $\left.10^{-4} \sqrt{(1-e) /(1+e)} / \sqrt{a_{\text {res }}[\mathrm{AU}]} \approx 1 \times 10^{-5}\right)$. Considering ideally spherical particles $\beta_{2} \equiv 0$. Nonsphericity of a particle is usually formulated in terms of the so-called aspect ratio (e.g. Grynko \& Shkuratov 2003) - it is the ratio of largest to smallest characteristic size of the particle. The larger the aspect ratio, the more evident the bimodality of the density function for $\beta_{2}$. In this sense our numerical results imply that spherical particles are preferrably captured at highly eccentric orbits, while nonspherical particles are predominately distributed along slightly eccentric (or circular) orbits. This is in good agreement with our previous results obtained by independent direct calculations realized for a set of single particles (Klačka \& Kocifaj 2006a). In particular, these simulations have shown that nonspherical particles captured in mean-motion resonances with a planet can be predominately found in orbits with small $e_{\text {in }}$.

Nevertheless, the nonspherical interplanetary dust particles moving in the Solar System can survive at highly eccentric orbits, too (Kocifaj et al. 2000). Contrary to Eq. (6) this situation corresponds to specific evolutionary conditions when $e \approx e_{\text {in }}$ 


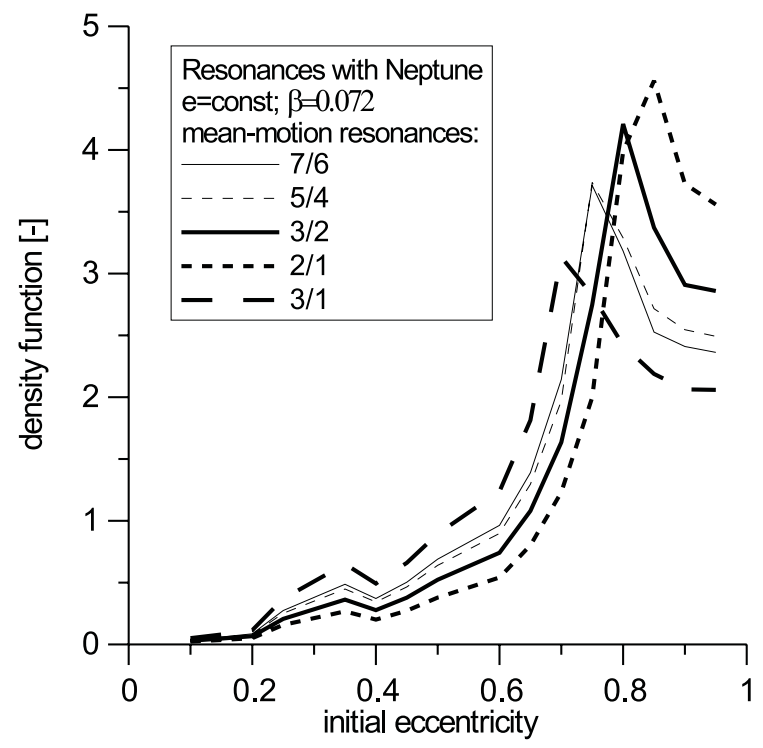

Fig. 5. The density function for $e(t)=e_{\text {in }}$, where initial eccentricity varies in the range $\langle 0.1,0.95\rangle$ to be consistent with Fig. 1 . Several meanmotion resonances are considered.

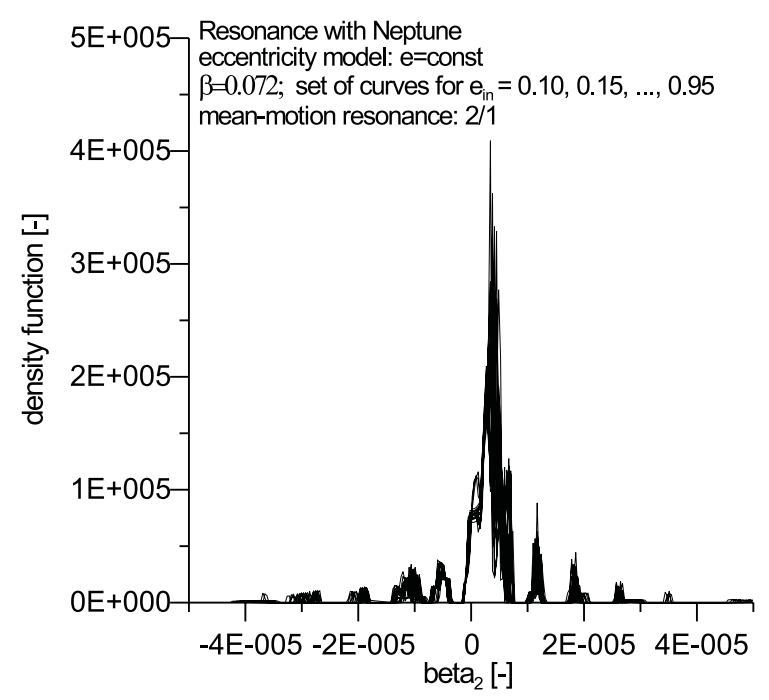

Fig. 6. The density function for $\beta_{2}$ with $e_{\text {in }} \in\langle 0.1,0.95\rangle$.

(see, e.g., Fig. 2 in Klačka \& Kocifaj 2001). Having this fact in mind, one may expect that nonspherical particles captured in mean-motion resonances can be also found at highly eccentric trajectories, but only when eccentricity is almost constant. Therefore we tried to repeat the entire set of calculations, however, assuming that $e(t)=e_{\text {in }}$. The density function for $e_{\text {in }}$ is depicted in Fig. 5. As predicted, the capture probability is quite small at circular orbits and it significantly increases as $e_{\text {in }}$ approaches $\approx 1$. The density function for mean-motion resonance $2 / 1$ peaks now at about $e_{\text {in }} \approx 0.9$. The profile of the density function for $\beta_{2}$ shows that particles with small aspect ratios are the best candidates to survive in mean-motion resonance $2 / 1$ (Fig. 6). However, the existence of subsidiary modes indicates that nonspherical particles with moderate aspect ratios could also temporarily remain in the resonance $2 / 1$. All curves for $e_{\text {in }}=$ $0.10,0.15, \ldots, 0.95$ are brought together in Fig. 6 , because the density functions for all studied initial eccentricities have similar shapes.

\section{Discussion}

We have found that the values of $\beta_{2}$ may be of the order of $10^{-4}$. The values of $\beta_{2} / \beta$ are much larger than the term $v / c$. Thus, the Poynting-Robertson effect is not a correct approximation for the orbital evolution of real, arbitrarily shaped dust grains. This result, obtained as a solution of the inverse task, is consistent with the direct calculation of the orbital evolution of nonspherical dust grains (Klačka et al. 2005; Klačka \& Kocifaj 2006a). The sign of $\beta_{2}$ is also important: plus and minus values show that the scattering is in opposite orientations of a given direction. Almost symmetric peaks in Figs. 2, 3 are in agreement with the stability of the particle in the mean-motion resonance: preferred either negative or positive values of $\beta_{2}$ would cause ejection of the particle from the resonance.

Real dust particles are nonspherical. Observations confirm this statement by measurements of polarization of the scattered light (Kimura \& Mann 2004; Jockers 1997; or Levasseur-Regourd et al. 1999): polarization at small scattering angles is negative. However, no discussion on polarization of the radiation is presented in papers confirming the existence of a dust ring around the Sun in the vicinity of the planets (as for the Earth, see Dermott et al. 1994; Brownlee 1994; Reach et al. 1995).

It seems that the larger the aspect ratio of the particle, the shorter the capture time in a resonance (Klačka et al. 2005). Knowing the total number of dust particles in a ring around the Sun, i.e. in the resonance, one could calculate the density function of the aspect ratio for an influx of interplanetary particles. The situation is in reality more complicated, since nonspherical particles can be captured even from the region with smaller semi-major axis than corresponding to the resonance; spherical grains can be captured only during the process of inspiralling toward the Sun. The analysis here is the inverse task to the problem solved in our preceding papers. However, dealing with the detailed influx of arbitrarily shaped dust grains, one has to solve direct task. But the numerical integration of the orbital evolution of a nonspherical particle (the direct task), without any symmetry in its shape, is computationally enormously time consuming.

Our results show that the first approximation of the "iterative" procedure used here (see text above Eq. (8)) yields results consistent with detailed numerical integrations realized as a solution of the direct task (Klačka \& Kocifaj 2006a). This simultaneously confirms the approach applied in this paper is relevant from a physical point of view.

\section{Conclusion}

We have simulated motion of dust particles under the action of the electromagnetic radiation of the Sun. Simultaneously, the gravity of the Sun and Neptune moving in a circular orbit around the Sun was considered. Specifically, for a single particle it represents a planar circular restricted three-body problem with the action of electromagnetic radiation. The paper shows that a prescribed orbital evolution of a particle near mean-motion orbital resonances with a planet can be understood as motion of a nonspherical particle with a nonzero nonradial component of the radiation pressure force, when viewed from the particle's frame of reference. The obtained results of the values of $\beta_{2}$ are consistent with detailed numerical simulations already published. Thus, the Poynting-Robertson effect cannot represent a complete physical result, since it states that $\beta_{2} \equiv 0$.

Acknowledgements. This work was partially supported by the Scientific Grant Agency VEGA, grant No. 1/3074/06. 


\section{References}

Beaugé, C., \& Ferraz-Mello, S. 1994, Icarus, 110, 239

Bohren, C. F., \& Huffman, D. R. 1983, Absorption and Scattering of Light by Small Particles (New York: John Wiley \& Sons, Inc.)

Brownlee, D. E. 1994, Nature, 369, 706

Dermott, S. F., Jayaraman, S., Xu, Y. L., Gustafson, B. Å. S., \& Liou, J. C. 1994, Nature, 369, 719

Einstein, A. 1905, Ann. Phys., 17, 891

Grynko, Ye., \& Shkuratov, Yu. 2003, J. Quant. Spectrosc. Rad. Transfer, 78, 319

Jackson, A. A., \& Zook, H. A. 1989, Nature, 337, 629

Jockers, K. 1997, Earth, Moon Planets, 79, 221

Kimura, H., \& Mann, I. 2004, J. Quant. Spectrosc. Rad. Transfer, 89, 155

Klačka, J. 1992, Earth, Moon, Planets, 59, 41

Klačka, J. 1993, Earth, Moon, Planets, 62, 239

Klačka, J. 1994, Earth, Moon, Planets, 64, 125

Klačka, J. 2000, http://xxx. lanl.gov/abs/astro-ph/0008510

Klačka, J. 2004, Cel. Mech. Dynam. Astron., 89, 1

Klačka, J., \& Kocifaj, M. 1994, Electromagnetic radiation and equation of motion for a dust particle. in Dynamics and Astrometry of Natural and Artificial Celestial Bodies, ed. K. Kurzynska, F. Barlier, P. K. Seidelmann, \& I. Wytrzyszczak (Poznan, Poland: Astronomical Observatory of A. Mickiewicz University), 187

Klačka, J., \& Kocifaj, M. 2001, J. Quant. Spectrosc. Rad. Transfer, 70, 595

Klačka, J., \& Kocifaj, M. 2006a, J. Quant. Spectrosc. Rad. Transfer, 100, 187

Klačka, J., \& Kocifaj, M. 2006b, Effect of Electromagnetic Radiation on Dynamics od Cosmic Dust Particles, in Space Science: New Research, ed. N. S. Maravell (New York: Nova Science Publishers, Inc.), 245
Klačka, J., Kocifaj, M., \& Kundracík, F. 2006, Planet. Space Sci., 54, 379 Klačka, J., Kocifaj, M., \& Pástor, P. 2005, J. Phys. Conf. Ser., 6, 126

Kocifaj, M., Klačka, J., \& Kundracík, F. 2000, Motion of realistically shaped cosmic dust particle in Solar System, in Light Scattering by Nonspherical Particles: Halifax Contributions, ed. G. Videen, Q. Fu, \& P. Chýlek (Adelphi Maryland, USA: Army Research Laboratory), 257

Krauss, O., \& Wurm, G. 2004, J. Quant. Spectrosc. Rad. Transfer, 89, 179

Leinert, Ch., \& Grûn, E. 1990, Interplanetary dust. in Physics of the Inner Heliosphere I, ed. R. Schwen, \& E. Marsch (Berlin: Spriger-Verlag), 207

Levasseur-Regourd, A. C., Cabane, M., \& Haudebourg, V. 1999, J. Quant. Spectrosc. Rad. Transfer, 63, 631

Liou, J.-Ch., \& Zook, H. A. 1995, Icarus, 113, 403

Liou, J.-Ch., \& Zook, H. A. 1997, Icarus, 128, 354

Liou, J.-Ch., Zook, H. A., \& Jackson, A. A. 1995, Icarus, 116, 186

Makuch, M., Brilliantov, N. V., Sremčević, M., Spahn, F., \& Krivov, A. V. 2006, Planet. Space Sci., 54, 855

Marzari, F., \& Vanzani, V. 1994, A\&A, 283, 275

Mie, G. 1908, Ann. Phys., 25, 377

Mishchenko, M., Travis, L. D., \& Lacis, A. A. 2002, Scattering, Absorption and Emission of Light by Small Particles (Cambridge: Cambridge University Press)

Reach, W. T., Franz, B. A., Welland, J. L., et al. 1995, Nature, 374, 521

Robertson, H. P. 1937, MNRAS, 97, 423

Šidlichovský, M., \& Nesvorný, D. 1994, A\&A, 283, 972

Weidenschilling, S. J., \& Jackson, A. A. 1993, Icarus, 104, 244

Wurm, G., \& Schnaiter, M. 2002, Fractal aggregates in space, in Optics of Cosmic Dust, ed. G. Videen, \& M. Kocifaj (Dordrecht, Boston, London: Kluwer Academic Publishers), 89 